\title{
Matrix interference in ligand-binding assays: challenge or solution?
}

\author{
"One of the key characteristics in ligand binding assays that requires detailed attention is the understanding of the \\ difference between selectivity (matrix effect) and specificity (cross-reactivity)."
}

Ligand binding assays (LBAs) still remain the workhorse for the quantification of biotherapeutics and biomarkers. These molecules include, but are not limited to, proteins, large peptides, soluble receptors, antibody-drug conjugates and oligonucleotides. Many of the molecules that are of therapeutic use are biologically derived, and the structure-function relationship can be unique and complex. Alternatively, those that are considered biomarkers can be bound to other molecules that serve a specific function. Hence, the accurate quantification of these molecules that impact the PK, PD, immunogenicity and efficacy data during drug development is critical.

One of the key characteristics in LBA that requires detailed attention is the understanding of the difference between selectivity (matrix effect) and specificity (cross-reactivity). The specificity, which is often confused and misinterpreted as selectivity in LBA, primarily relates to the unique reagents that are used in the assays [1]. Selectivity, or matrix effect, is the interference in the assay that results from the various components of the matrix. These can be specific or nonspecific in nature. Specific matrix effects can occur when a matrix component, that is, an endogenous molecule, similar to the analyte can interfere in the assay. Nonspecific matrix effects can be from other matrix components that interfere in the assay $[2,3]$.

Overcoming matrix effect is a critical method development task for any LBA so that a robust and rugged method can be validated to support drug development.

This themed issue contains a review, research articles and short communications that describe a variety of strategies to understand and overcome matrix effects in LBAs using different methods or platforms [4-15].

The three editorials in this issue by Lee, Wang and Ma, and Crisino et al., have described how orthogonal evaluations, multiple method development, reagent screening and emerging technology strategies could be used to either minimize or overcome matrix effects in the assays. The advancement of LC-MS for the quantification of large molecules has provided the bioanalytical scientists with orthogonal methods and additional tools to use to address matrix effects [16]. In addition, the Lee editorial describes different considerations that need to be evaluated before finalizing the methodology, such as what is being measured (Free/Total), the biotransformation of the compound, the available technologies and so on.

The article by Wang and Ma, while calling matrix effects the Achilles heel of the LBA, provides a good overview of a variety of strategies that one can investigate to minimize or eliminate matrix effects.

Crisino et al., while reporting the active work of one of the teams of the AAPS Emerging Technology Group, address some of the newer technologies that are emerging, which may have the potential to reduce and eliminate matrix effects. The authors forewarn scientists that while new technologies may not be the magic bullet to resolving matrix issues, they provide an alternative approach to solving some of the main issues. The authors have done a good job describing the technologies and providing the basic background for some of the considerations.

Gorovitz et al. review the many factors that affect matrix interference in LBA in their article. The authors provide practical tips for reducing these effects for the development and validation of successful assays.

Shih et al. describe a complete and comprehensive multiprong approach to solving issues with matrix effects. These include: reagent screening and selection; using orthogonal methods, specifically bio-layer interferometry, to identify the source of the matrix effect; using design of experiment strategies to understand the interdependencies; and finally, describing a case study that encompassed these techniques

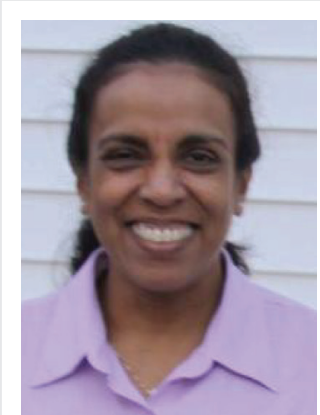

Binodh DeSilva

Author for correspondence:

Bristol Myers Squibb, Route 206

\& Provinceline Road, Princeton, NJ 08543 , USA

Tel.: +I 6092524274

binodh.desilva@bms.com

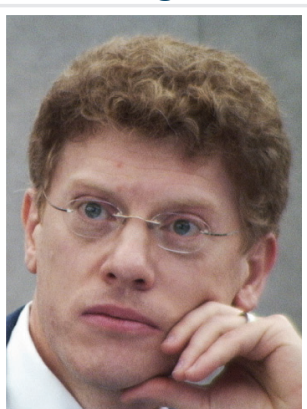

Fabio Garofolo

Algorithme Pharma Inc., 575 Armand-Frappier, Laval, Quebec, H7V 4B3, Canada 
to successfully validate a method for drug development use.

Myler et al. describe a real-life example in the era of global clinical studies and multiple laboratories supporting the bioanalytical efforts. In this article, they describe how a cross validation was implemented to bridge the assays so that data from these various laboratories were equivalent to support regulatory submissions. As described, matrix effect was critical to the bridging and how the matrix pool had to be designated as a 'critical reagent' in these assays. In addition, this example clearly demonstrates the importance of reagents, matrix and other components of a LBA during lifecycle management.

The article by Yang et al. complements the Crisino et al. editorial where they describe the use of a newer technology, the Gyrolab ${ }^{\mathrm{TM}}$ immunoassay platform, which adapts micro fluidic systems that promote fast assay kinetics with shorter contact time between analytes, enabling the reduction of matrix interferences in the assays. This technique also uses nanoliter scale volumes and allows faster diffusion times, as well as enabling the use of this technology for rodent studies where the volume of sample can be limited.

"Understanding the basis of systems biology,
the physiochemical/biochemical properties of
the analytes and the specificity of the reagents,
all the parameters that can potentially impact
the ligand binding assay, and mainly using
good science, is crucial to successfully
overcoming the matrix effect."

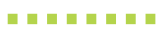

The article by Chilewski et al. also describes the Gyrolab platform and its use in helping address the matrix interference when assays are transferred from a preclinical stage to the clinical stage, where the sensitivity requirements necessitate a further reduction of the matrix interferences.

Spriggs et al. describe two case studies; strategies to resolve specific and nonspecific matrix effects. The advantage of using design of experiments is further illustrated in one of the examples. While the determination of antidrug antibody (immunogenicity) assays are qualitative in nature, one of the examples in this article clearly demonstrates the value in assessing matrix effects even in these situations to understand and deliver reliable data in drug development.
There are three articles describing the importance of resolving matrix interference in biomarker assays (Zhao et al., Fraser and Soderstrom, and Thway and Salimi-Moosavi). Most biomarkers have an endogenous counterpart in the matrix, and often the levels of the endogenous analytes differ based on the disease population, stage and even environmental condition. Other nonspecific matrix interferences may also cause interference in these assays. While there is a strong desire to minimize the effects of pre-analytical and laboratory processes on the quantifiable levels, and these are not specifically covered here, these factors also play a major role during the analysis of biomarkers [16].

Thway and Salimi-Moosavi describe two case studies where orthogonal methods were used to investigate matrix effects. In addition to this, these case studies also demonstrate the impact of the quality and identity of the reference material used in the assays.

The criticality of evaluating matrix effects when measuring ultra-low, sub-pg/ml, biomarker concentrations is described in the article from Fraser and Soderstrom. The primary impact of matrix interference on this IL-13 assay was to increase the LLOQ from the previously reported $0.07-0.3 \mathrm{pg} / \mathrm{ml}$. They have shown that the use of surrogate recombinant version of the IL-13 molecule does not provide accurate data and does not represent the endogenous molecule. Therefore, the researchers have used incurred samples to establish the stability of the biomarker.

The Zhao et al. article reports the optimization and fit-for-purpose validation of an ultra-sensitive electrochemiluminescence assay for thymus and activation-regulated chemokine (TARC) quantitation in human plasma. In this article, they describe the evaluation of different buffer systems to eliminate matrix interference and to be able to quantify the biomarker post-treatment [16].

The themed issue on resolving matrix effect in LBA has a collection of articles with diverse options and strategies from a variety of experts in the field. As technology evolves, there will be numerous further efforts to reduce this very important assay variable. Understanding the basis of systems biology, the physiochemical/biochemical properties of the analytes and the specificity of the reagents, all the parameters that can potentially impact the LBA, and mainly using good science, is crucial to successfully overcoming the matrix effect. The overall key message is that it is vital to understand the impact of matrix effect in 
LBA and communicate the data based on that to the stakeholders.

\section{Financial \& competing interests disclosure}

The authors have no relevant affliations or financial involvement with any organization or entity with a financial interest in or financial conflict with the subject matter or materials discussed in the manuscript. This includes employment, consultancies, honoraria, stock ownership or options, expert testimony, grants or patents received or pending, or royalties. No writing assistance was utilized in the production of this manuscript.

\section{References}

1 Rup B, O'Hara D. Critical ligand binding reagent preparation/selection: when specificity depends on reagents. AAPS J. 9, E148-E155 (2007).

2 DeSilva B, Smith W, Weiner R et al. Recommendations for the bioanalytical method validation of ligand-binding assays to support pharmacokinetic assessments of macromolecules. Pharm. Res. 11, 1885-1900 (2003).

3 Kelley M, DeSilva B. Analytical considerations for immunoassays for macromolecules. In: Handbook of Pharmaceutical Biotechnology. Cox Gad S (Ed.). Wiley-InterScience, John Wiley \& Sons Inc., Hoboken, NJ, USA (2007).

4 Gorovits B, McNally J, Fiorotti C, Leung S. Protein-based matrix interferences in ligand-binding assays. Bioanalysis 6(8), 1131-1140 (2014).

5 Thway T, Salimi-Moosavi H. Evaluating the impact of matrix effects on biomarker assay sensitivity. Bioanalysis 6(8), 1081-1091 (2014).

6 Spriggs FP, Duriga N, Rathi A, Qu Q. Resolution of matrix interference: quantitative and quasi-quantitative ligand-binding assays case studies. Bioanalysis 6(8), 1093-1101 (2014).

7 Shih JY, Patel V, Ma M. Strategic approaches for assessment and minimization of matrix effect in ligand-binding assays. Bioanalysis 6(8), 1103-1112 (2014).

8 Myler H, Felix T, Zhu J, Hruska M, Piccoli SP. Measuring biotherapeutics with endogenous counterparts and pre-existing antibodies: an interferon case study. Bioanalysis 6(8), 1113-1122 (2014).

9 Crisino RM, Luo L, Geist B, Zoghbi J, Spriggs F. Matrix effect in ligand-binding assay: the importance of evaluating emerging technologies. Bioanalysis 6(8), 1033-1036 (2014).

10 Lee JW. Orthogonal tools to help determine the required selectivity of ligand-binding assays in drug development. Bioanalysis 6(8), 1037-1040 (2014).

11 Wang Q, Ma M. Are matrix effects the Achilles heel in the bioanalysis of biotherapeutics by ligand-binding assays? Bioanalysis 6(8), 1041-1044 (2014).

12 Yang T-Y, Uhlinger DJ, Ayers SA, O'Hara DM, Joyce AP. Challenges in establishing selectivity, specificity and quantitation range of ligand-binding assays: case studies with a microfluidic immunoassay platform. Bioanalysis 6(8), 1049-1057 (2014).

13 Chilewski SD, Mora JR, Gleason C, DeSilva B. Addressing matrix effects in ligandbinding assays through the use of new reagents and technology. Bioanalysis 6(8), 1059-1067 (2014).

14 Zhao X, Delgado L, Weiner R, Laterza OF. An ultra-sensitive clinical biomarker assay: quantitation of thymus and activationregulated chemokine in human plasma. Bioanalysis 6(8), 1069-1080 (2014).

15 Fraser S, Soderstrom C. Due diligence in the characterization of matrix effects in a total IL-13 Singulex ${ }^{\mathrm{TM}}$ method. Bioanalysis 6(8), 1123-1129 (2014).

16 Lee JW, Devanarayan V, Barrett YC et al. Fit-for-purpose method development and validation for successful biomarker measurement. Pharm. Res. 23, 312-328 (2006). 